\title{
Thermodynamic Limit for Classical Systems with Coulomb Interactions in a Constant External Field
}

\author{
O. Penrose and E. R. Smith \\ Mathematics Department, The Open University, Walton Hall, Walton, Bletchley, \\ Bucks, U. K.
}

Received November 16, 1971, in revised form January 11, 1972

\begin{abstract}
We introduce a new method for studying the thermodynamic limit for systems of particles with Coulomb interactions. The method is based on calculating the potential energy of the Coulomb interactions from the electric or magnetic fields in the system rather than from the energy of the individual particle - particle interactions. We are able to include the effects of a constant external field being imposed at the boundary of the system. The difficulties associated with Coulomb potentials being not even weakly tempered are overcome by imposing the boundary condition that at the boundary of the region containing the particles, the electric or magnetic field has normal component equal to that of the applied field. We prove that the thermodynamic free energy density exists and is independent of the sequence of regions used to define the limit. We introduce sequences of regions all of the same shape and show that for these sequences of regions the thermodynamic free energy density is independent of shape. Finally, we prove that the thermodynamic free energy is a convex function of the density of particles and of the applied field.
\end{abstract}

\section{Introduction}

In this paper we describe a new method for proving the existence of thermodynamic functions for systems where the interactions between the particles or molecules of the system include long range Coulomb forces and where in addition external electric or magnetic fields may be present. The interactions we consider include the forces between magnetic dipoles in paramagnets and ferromagnets, the forces between electric dipoles in dielectrics and the forces between charged particles in ionic systems. However, we specifically exclude from consideration interactions attributable to the interference of electric and magnetic fields, such as the interactions of charged particles with magnetic fields produced by electric currents; in consequence our treatment does not apply to diamagnetic substances. In addition to proving the existence of the free energy for such systems, we discuss rigorously some of the simple properties of the free energy. The method also makes it possible to include in 
the rigorous discussion the shape dependent effects that are, experimentally, a characteristic feature of the thermodynamic functions of systems with Coulomb interactions. We leave a discussion of these effects to a later paper.

For systems with short range forces only, the best method of proving the existence of the thermodynamic limit is the one originated by Ruelle [1]. He considered a system of $N$ particles whose positions we denote by $\boldsymbol{x}_{1}, \boldsymbol{x}_{2}, \ldots, \boldsymbol{x}_{N}$, interacting by two body forces with a potential $\varphi(r)$ satisfying the two conditions

(i) Stability:

where

$$
u<\infty
$$

$$
-u \equiv \inf _{N \geqq 1} \frac{1}{N} \inf _{\boldsymbol{x}_{1}, \boldsymbol{x}_{2}, \ldots, \boldsymbol{x}_{N}} \sum_{1 \leqq i<j \leqq N} \varphi\left(\left|\boldsymbol{x}_{i}-\boldsymbol{x}_{j}\right|\right)
$$

is the greatest lower bound on the mean energy per particle. Physically this condition ensures that the system of particles does not collapse in on itself.

(ii) Strong tempering: for some $R_{0}<\infty$

$$
\varphi(r) \leqq 0 \quad \text { for all } r \geqq R_{0} .
$$

Physically this second condition ensures that the system of particles does not disperse.

The stability condition has the consequence that $Z(N, \Omega)$, the classical partition function for $N$ particles in a region $\Omega$ has the upper bound

$$
Z(N, \Omega) \leqq\left(\lambda e^{\beta u}|\Omega|\right)^{N} / N \text { ! }
$$

where $\lambda \equiv\left(2 \pi m / \hbar^{2} \beta\right)^{3 / 2}, \beta \equiv 1 / k T$, which we shall treat as a constant in this paper and $|\Omega|$ denotes the volume of the region $\Omega$. The strong tempering condition has the consequence that if $\Omega^{\prime}$ and $\Omega^{\prime \prime}$ are two regions whose separation is at least $R_{0}$ and if $\left(\Omega^{\prime} \cup \Omega^{\prime \prime}\right) \subset \Omega$, then

$$
Z\left(N^{\prime}+N^{\prime \prime}, \Omega\right) \geqq Z\left(N^{\prime}, \Omega^{\prime}\right) Z\left(N^{\prime \prime}, \Omega^{\prime \prime}\right) .
$$

Ruelle used the inequalities (1.3) and (1.4) to show that

$$
\lim _{N \rightarrow \infty}\left[\log Z\left(N, \Omega_{N}\right)\right] / N
$$

exists where $\Omega_{1}, \Omega_{2}, \ldots$ is a sequence of cubes whose volumes satisfy

$$
\lim _{N \rightarrow \infty}\left|\Omega_{N}\right| / N=v
$$

and $v$, the specific volume, may have any value greater than the specific volume at close packing. Fisher [2] extended Ruelle's proof to regions of 
more general shape and also showed that the condition (1.2) may be weakened to

(ii') Weak tempering: for some $R_{0}<\infty$

$$
\varphi(r) \leqq D_{2} / r^{3+\varepsilon} \quad \text { for } \quad r \geqq R_{0}
$$

where $D_{2}$ and $\varepsilon$ are positive constants.

For classical system with Coulomb as well as strong enough short range repulsive forces it is possible to prove stability without too much difficulty $[3,4]$, but without the short range repulsive forces, a system of classical particles with Coulomb (const/r) interactions is not stable in the sense of (1.1). A system of quantum mechanical particles with only Coulomb interactions is stable if a large enough proportion of them are Fermions [5] because the Pauli exclusion principle then takes the place of the short range repulsive forces. It should be noted in this context that classical stability implies quantum mechanical stability but not vice versa.

However, for any system with Coulomb as well as other forces, even the weakened form of the tempering condition (1.7) is violated, both for charged particles (since $\varphi(r)=$ (const) $/ r>0$ for particles of like charge) and for dipoles (since $\varphi(r)=$ (const) $/ r^{3}>0$ for similarly directed collinear dipoles).

Lebowitz and Lieb [6] have examined the problem for a system of point charges with almost zero total charge in zero external field. To obtain an inequality of the type (1.4) they use a symmetrization technique to allow for the screening of the charges in the system by other oppositely charged particles, so that the average interaction of a particle with the rest of the system is tempered strongly enough.

The intention of the present paper is to provide a proof that the thermodynamic limit exists which applies even if an external field is present. For this case the symmetrization arguments used by Lebowitz and Lieb no longer apply. Our method is to replace the tempering condition by a special boundary condition on the electric and magnetic fields in the system; this boundary condition makes it possible to deduce (1.4) even though the Coulomb potential violates the tempering condition.

Basically, the special boundary condition is that the normal components of the electric and magnetic field at the surface of the container must vanish. For the magnetic field, this boundary condition has the interpretation that the material of the container walls is an ideal superconductor. For the electric field, the best available physical interpretation is that the material has a vanishing dielectric constant (in contrast to the hypothetical fluid of infinite dielectric constant used by Onsager [3] in his proof of stability). To keep the physical interpretation simple, we 
shall mainly consider a system of magnetic dipoles. The generalization to a mixture of electric charges and dipoles will be discussed later.

A preliminary outline of this work was given at the I.U.P.A.P. Conference on Statistical Mechanics in 1966 [11].

\section{Description of the System}

We restrict the detailed presentation to a pure (one component) classical system with short range forces and magnetostatic forces, for example a system of magnetic dipoles. We include in the short range forces those due to the particles in the system having a hard spherical core of finite radius. We denote the number of particles or molecules in the system by $N$.

We assume that the Hamiltonian has the form

$$
\mathscr{H}=K+U+W
$$

where $K$ denotes the kinetic energy, $U$ the potential energy due to the short range forces and $W$ the energy due to the magnetic field. The system is classical so that the kinetic energy is given by

$$
K=\sum_{i=1}^{N} K_{i}=\sum_{i=1}^{N} K_{(1)}\left(\boldsymbol{p}_{i}, \boldsymbol{q}_{i}\right)
$$

where $\boldsymbol{q}_{i}$ represents the position coordinates of the $i^{\text {th }}$ molecule and may include, as well as the three coordinates of the centre of mass of the $i^{\text {th }}$ molecule, the angle and internal coordinates of that molecule. The vector $\boldsymbol{p}_{i}$ represents the generalized momentum of the $i^{\text {th }}$ molecule. $K_{(1)}$ is the single molecule kinetic energy and is a positive definite quadratic form:

$$
K_{(1)}(\boldsymbol{p}, \boldsymbol{q})=\frac{1}{2} \sum_{k} \sum_{l} a_{k, l}(\boldsymbol{q}) p_{k} p_{l}
$$

where $p_{k}$ and $p_{l}$ are components of $\boldsymbol{p}$.

For the short range potential we assume the form

$$
U=\sum_{1 \leqq i<j \leqq N} U_{i, j}+\sum_{1 \leqq i \leqq N} U_{i}
$$

where $U_{i, j}$ is the potential energy of the interaction between the $i^{\text {th }}$ and $j^{\text {th }}$ molecules by short range forces and $U_{i}$ is the internal potential energy of the $i^{\text {th }}$ molecule plus the potential energy of the interaction between the molecule and the container of the system. We assume the two body potential energy to have the form

$$
U_{i, j}=U_{(2)}\left(\boldsymbol{q}_{i}, \boldsymbol{q}_{j}\right)
$$


where $U_{(2)}$ is symmetric and translationally invariant. We also assume that $U_{(2)}$ satisfies

(a) the stability condition $u_{(2)}<\infty$ where

$$
-u_{(2)} \equiv \inf _{N} N^{-1} \inf _{\boldsymbol{q}_{1} \ldots \boldsymbol{q}_{N}} \sum_{1 \leqq i<j \leqq N} U_{i, j}
$$

and (b) the strong tempering condition

$$
U_{i, j} \leqq 0 \quad \text { if } \quad\left|\boldsymbol{r}_{i}-\boldsymbol{r}_{j}\right| \geqq R_{0} \quad \text { for some } \quad R_{0}<\infty
$$

where $\boldsymbol{r}_{i}$ is the position vector of the centre of mass of the $i^{\text {th }}$ molecule with position coordinates $\boldsymbol{q}_{i}$.

The one-particle potential $U_{(1)}$ arises from two separate physical processes: the internal forces between one part of the molecule and another and the external forces (other than magnetic ones) between the molecule and the wall. We assume that $U_{(1)}$ is bounded below and that the forces between the molecules and the container are strong enough to ensure that the entire magnetic moment carried by the molecules remains inside the region $\Omega$ enclosed by the container. That is, we assume

(a) $U_{(1)}(q) \geqq-u_{(1)}$

(b) if $U_{(1)}(\boldsymbol{q})<+\infty$ then $\boldsymbol{m}(\boldsymbol{x}, \boldsymbol{q})=0$ for all $\boldsymbol{x} \notin \Omega$

where $\boldsymbol{m}(\boldsymbol{x}, \boldsymbol{q})$ is the magnetic moment density (or intensity of magnetization) at the point $\boldsymbol{x}$ produced by a molecule with position $\boldsymbol{q}$.

For example, if the molecules are uniformly magnetized hard spheres of radius $a / 2$, we may take $U_{(1)}(\boldsymbol{q})$ to be zero if the distance from $\boldsymbol{r}$ to the complement of $\Omega$ exceeds $a / 2$ and to be $+\infty$ if not. It is useful to assume that $R_{0}>a / 2$, which we can arrange by changing $R_{0}$ in (2.7).

For the contribution to the potential energy from the magnetostatic interactions, we use the formula

$$
W=(1 / 8 \pi) \int_{\Omega} \boldsymbol{H}^{2}(\boldsymbol{x}) d^{3} \boldsymbol{x}
$$

where $\boldsymbol{H}$ is the magnetic field strength in e.m.u. The integration is confined to the region $\Omega$ because we are not interested here in the energy of the boundary material, nor of anything outside the boundary. The field $\boldsymbol{H}$ is to be calculated from Maxwell's equations, which in the static (current free) approximation used in this paper reduce to

and

$$
\nabla \times \boldsymbol{H}(\boldsymbol{x})=0
$$

where

$$
\nabla \cdot[H(x)+4 \pi \boldsymbol{m}(\boldsymbol{x})]=0
$$

$$
\boldsymbol{m}(\boldsymbol{x}) \equiv \sum_{i=1}^{N} \boldsymbol{m}\left(\boldsymbol{x}, \boldsymbol{q}_{i}\right)
$$


is the total magnetic moment density at the point $\boldsymbol{x}$. We shall require $\boldsymbol{m}(\boldsymbol{x}, \boldsymbol{q})$ to be, for every fixed $\boldsymbol{q}$, a continuously differentiable function of $\boldsymbol{x}$ except on a finite number of smooth surfaces, at which it may be discontinuous. This requirement excludes point dipoles (such as a spinning electron) from the treatment, but allows them to be represented approximately by small magnetized spheres.

As indicated in the introduction, the basic idea of this paper is to replace the tempering conditions on the potential normally used in thermodynamic existence proofs by a boundary condition requiring that the normal component of $\boldsymbol{H}$ vanish on $\partial \Omega$, the boundary of $\Omega$. This boundary condition corresponds to a superconducting wall which shields the system from an external field. It is quite easy and later very useful to generalize the method so that the effects of constant external fields on the system may be considered. To achieve this we use the more general boundary condition

$$
\boldsymbol{H}_{n}(\boldsymbol{x})=\boldsymbol{H}_{0, n}(\boldsymbol{x}) \quad \text { if } \quad \boldsymbol{x} \in \partial \Omega
$$

where $\boldsymbol{H}_{n}$ denotes the normal component of $\boldsymbol{H}$ and $\boldsymbol{H}_{0, n}$ denotes the normal component of the constant external field $\boldsymbol{H}_{0}$. We assume that the surface $\partial \Omega$ is smooth so that we have a suitable interpretation of the normal to $\partial \Omega$. This more general boundary condition allows the physical interpretation that $\boldsymbol{H}_{0}$ is a uniform field frozen into the superconducting boundary layer.

We now verify that the differential Eqs. (2.10) and (2.11), taken together with the boundary condition (2.13), do specify a unique field $\boldsymbol{H}(\boldsymbol{x})$ in $\Omega$. Let $X_{1}(\boldsymbol{x})$ denote the magnetic scalar potential produced in infinite space by the magnetic moment distribution $\boldsymbol{m}(\boldsymbol{x})$. It is given by the Poisson integral

where

$$
\begin{aligned}
X_{1}(\boldsymbol{x}) & =\int \boldsymbol{m}\left(\boldsymbol{x}^{\prime}\right) \cdot \nabla\left(\left|\boldsymbol{x}-\boldsymbol{x}^{\prime}\right|\right)^{-1} d^{3} \boldsymbol{x}^{\prime} \\
& =\int \sigma\left(\boldsymbol{x}^{\prime}\right) /\left(\left|\boldsymbol{x}-\boldsymbol{x}^{\prime}\right|\right) d^{3} \boldsymbol{x}^{\prime} \\
\sigma(x) & =-\nabla \cdot \boldsymbol{m}(\boldsymbol{x})
\end{aligned}
$$

is Poisson's equivalent pole distribution. The distribution $\sigma$ is a continuous volume distribution in $\Omega$ except on the surface of discontinuity of $\boldsymbol{m}(\boldsymbol{x})$, where $\sigma$ is a continuous surface distribution.

The potential $X_{1}(x)$ is continuous ([7], p. 246-248) and its first and second derivatives are also continuous except on those surfaces on which $\boldsymbol{m}(\boldsymbol{x})$ is not continuous. On these surfaces the normal component of $\nabla X_{1}(x)$ may have a finite jump, but the tangential component remains continuous. The potential $X_{1}$ satisfies Poisson's equation inside $\Omega$ :

$$
\nabla^{2} X_{1}(x)=4 \pi \sigma(x)
$$


where we interpret both sides of (2.16) appropriately on the surfaces of discontinuity of $\boldsymbol{m}(\boldsymbol{x})$. By (2.15), $\boldsymbol{H}_{1}(\boldsymbol{x}) \equiv \nabla X_{1}(\boldsymbol{x})$ satisfies the differential Eqs. (2.10) and (2.11) but not the boundary condition (2.13).

In order to obtain a magnetic field $\boldsymbol{H}$ which satisfies both the pair of Eqs. (2.10) and (2.11) and the boundary condition (2.13), we introduce a second potential $X_{2}(x)$ satisfying Laplace's equation

$$
\nabla^{2} X_{2}(x)=0 \text { for } x \in \Omega
$$

and satisfying the boundary condition

$$
\frac{\partial \boldsymbol{X}_{2}}{\partial n} \equiv \boldsymbol{H}_{0, n}-\frac{\partial \boldsymbol{X}_{1}}{\partial n}
$$

for $x \in \partial \Omega$ with $\frac{\partial}{\partial n}$ denoting a derivative along the normal to $\partial \Omega$. For this Neumann problem to have a solution, a necessary condition is ([8], p. 230)

$$
\int_{\partial \Omega}\left(\boldsymbol{H}_{0, n}-\frac{\partial X_{1}}{\partial n}\right) d^{2} \boldsymbol{x}=0 .
$$

By virtue of Gauss's theorem and (2.11) and (2.16), this condition is satisfied. The condition (2.19) is also sufficient if the surface $\partial \Omega$ has a continuous second derivative ([8], p. 366). The function $X_{2}$ so defined is unique up to an arbitrary constant. Thus there exists a unique field

$$
\boldsymbol{H}(\boldsymbol{x})=\nabla\left(X_{1}+X_{2}\right)
$$

satisfying (2.10), (2.11) and (2.13) if $\partial \Omega$ is a surface with a continuous second derivative.

In most calculations in statistical mechanics, the potential energy is calculated as a sum of potential energies of one- and two-particle potentials, and the energy of interaction of a system with an external field $\boldsymbol{H}_{0}$ is written as $-\boldsymbol{H}_{0} \cdot \boldsymbol{M}$, where $\boldsymbol{M}$ is the total magnetic moment of the system. We now examine the connection between our potential energy expression (2.9) and the more usual way of writing down the potential energy. To this end we define

$$
\boldsymbol{H}_{1} \equiv \nabla X_{1} \quad \text { and } \quad \boldsymbol{H}_{2} \equiv \nabla X_{2}-\boldsymbol{H}_{0}
$$

so that using $(2.20)$ we have

$$
\begin{aligned}
\frac{1}{8 \pi} \int_{\Omega} \boldsymbol{H}^{2} d^{3} \boldsymbol{x}= & \frac{1}{8 \pi} \int_{\Omega} \boldsymbol{H}_{0}^{2} d^{3} \boldsymbol{x}+\frac{1}{4 \pi} \int_{\Omega} \boldsymbol{H}_{0} \cdot\left(\boldsymbol{H}_{1}+\boldsymbol{H}_{2}\right) d^{3} \boldsymbol{x} \\
& +\frac{1}{8 \pi} \int_{\Omega}\left(\boldsymbol{H}_{1}+\boldsymbol{H}_{2}\right)^{2} d^{3} \boldsymbol{x} .
\end{aligned}
$$


The first integral on the right of (2.21) is the vacuum energy of the external magnetic field $\boldsymbol{H}_{0}$. The second integral may be transformed in the following way. We use Cartesian coordinates $(x, y, z)$ with the $x$ axis along $\boldsymbol{H}_{0}$ so that

$$
\boldsymbol{H}_{0}=\left|\boldsymbol{H}_{0}\right| \nabla x
$$

and then, using Green's theorem, the definitions of $\boldsymbol{H}_{1}$ and $\boldsymbol{H}_{2}$ and (2.11), we obtain

$$
\begin{aligned}
\int_{\Omega} \boldsymbol{H}_{0} \cdot\left(\boldsymbol{H}_{1}+\boldsymbol{H}_{2}\right) d^{3} \boldsymbol{x} & =\left|\boldsymbol{H}_{0}\right| \int_{\Omega} \nabla x \cdot\left(\boldsymbol{H}-\boldsymbol{H}_{0}\right) d^{3} \boldsymbol{x} \\
& =4 \pi\left|\boldsymbol{H}_{0}\right| \int_{\Omega} x \nabla \cdot \boldsymbol{m}(\boldsymbol{x}) d^{3} \boldsymbol{x}
\end{aligned}
$$

where the surface integral from the application of Green's theorem vanishes by (2.13). We may apply Green's theorem again to the second line of (2.22) and, noting that $\boldsymbol{m}(\boldsymbol{x})=0$ for $\boldsymbol{x} \in \partial \Omega$, obtain

$$
\begin{aligned}
\frac{1}{4 \pi} \int_{\Omega} \boldsymbol{H}_{0} \cdot\left(\boldsymbol{H}_{1}+\boldsymbol{H}_{2}\right) d^{3} \boldsymbol{x} & =-\boldsymbol{H}_{0} \cdot \int_{\Omega} \boldsymbol{m}(\boldsymbol{x}) d^{3} \boldsymbol{x} \\
& =-\boldsymbol{H}_{0} \cdot \boldsymbol{M},
\end{aligned}
$$

where $\boldsymbol{M} \equiv \int_{\Omega} \boldsymbol{m}(\boldsymbol{x}) d^{3} \boldsymbol{x}$ is the total magnetic moment of the system. The second line of (2.23) gives an energy of exactly the type usually used in thermodynamics and in the statistical mechanics of magnetic lattice models for the interaction of a system with an external field.

We now examine the third integral on the right hand side of (2.21). Using Green's theorem we write

$$
\begin{aligned}
\int_{\Omega}\left(\boldsymbol{H}_{1}+\boldsymbol{H}_{2}\right)^{2} d^{3} \boldsymbol{x} & =\int_{\Omega}\left(\boldsymbol{H}_{1}+\boldsymbol{H}_{2}\right) \cdot \nabla\left(\boldsymbol{X}_{1}+\boldsymbol{X}_{2}-x\left|\boldsymbol{H}^{0}\right|\right) d^{3} \boldsymbol{x} \\
& =-\int_{\Omega} \nabla \cdot\left(\boldsymbol{H}_{1}+\boldsymbol{H}_{2}\right)\left(\boldsymbol{X}_{1}+\boldsymbol{X}_{2}-x\left|\boldsymbol{H}_{0}\right|\right) d^{3} \boldsymbol{x},
\end{aligned}
$$

the surface terms vanishing because $\boldsymbol{H}_{1, n}+\boldsymbol{H}_{2, n}=0$ on the surface by (2.13). Substituting (2.14) in (2.24) and using (2.11) we obtain

$$
\begin{aligned}
\frac{1}{8 \pi} \int_{\Omega}\left(\boldsymbol{H}_{1}+\boldsymbol{H}_{2}\right)^{2} d^{3} \boldsymbol{x}= & -\frac{1}{2} \int_{\Omega} \int_{\Omega} \frac{\sigma(\boldsymbol{x}) \sigma\left(\boldsymbol{x}^{\prime}\right)}{\left|\boldsymbol{x}-\boldsymbol{x}^{\prime}\right|} d^{3} \boldsymbol{x} d^{3} \boldsymbol{x}^{\prime} \\
& -\frac{1}{2} \int_{\Omega} \sigma(\boldsymbol{x})\left(X_{2}(\boldsymbol{x})-x\left|\boldsymbol{H}_{0}\right|\right) d^{3} \boldsymbol{x} .
\end{aligned}
$$

The first integral on the right hand side of (2.25) is the normal "Coulomb" energy of the system of molecules. The equivalent pole distribution $\sigma(x)$ may be written as a sum of contributions from individual molecules. This integral then includes the magnetic self-energies of the molecules and the usual molecule-molecule interaction terms. The magnetic self-energies 
of the molecules are finite because of condition (2.15), but they would not be finite for point charges.

The second integral on the right side of $(2.25)$ we regard as a surface term. The justification for this is that $\boldsymbol{H}_{2} \simeq 0$ in the deep interior of $\Omega$, so that we can choose the constant in its potential $\boldsymbol{X}_{2}(\boldsymbol{x})-\boldsymbol{x}\left|\boldsymbol{H}_{0}\right|$ in such a way that this potential is $\simeq 0$ in the deep interior of $\Omega$. We can interpret this surface term as giving the interaction of the charges, or magnetic poles, on the molecules with their images on the other side of the wall. Note that we may observe such forces experimentally when a permanent magnet floats above a superconducting disc.

\section{The Basic Lemma}

The main reason for adopting the boundary conditions (2.13) in this work is that they lead to the following lemma. Its physical interpretation is that the free energy of two subsystems isolated from one another by superconducting walls is decreased if the superconducting wall separating them is removed.

Lemma 1. Let $\Omega^{\prime}$ and $\Omega^{\prime \prime}$ be two non-overlapping regions with boundaries which have continuous second derivatives and let $\Omega$ be another such region containing both $\Omega^{\prime}$ and $\Omega^{\prime \prime}$. If $\boldsymbol{m}^{\prime}(\boldsymbol{x})$ and $\boldsymbol{m}^{\prime \prime}(\boldsymbol{x})$ are two magnetic moment densities which vanish outside $\Omega^{\prime}$ and $\Omega^{\prime \prime}$ respectively, with energies $W^{\prime}$ and $W^{\prime \prime}$ inside $\Omega^{\prime}$ and $\Omega^{\prime \prime}$ when a constant external field $\boldsymbol{H}_{0}$ is imposed at the surface of $\Omega^{\prime}$ and $\Omega^{\prime \prime}$, then the energy $W$ of the magnetic moment density $\boldsymbol{m}^{\prime}(\boldsymbol{x})+\boldsymbol{m}^{\prime \prime}(\boldsymbol{x})$ in the region $\Omega$ with a constant field $\boldsymbol{H}_{0}$ imposed at its surface satisfies

$$
W \leqq W^{\prime}+W^{\prime \prime}+\frac{\boldsymbol{H}_{0}^{2}}{8 \pi}\left(|\Omega|-\left|\Omega^{\prime}\right|-\left|\Omega^{\prime \prime}\right|\right) .
$$

To prove this lemma, we first convert the definition of $W$ given in Section II into a variational principle. Let $\mathscr{C}$ denote the class of vector fields $\boldsymbol{H}(\boldsymbol{x})$ which (i) are continuous and differentiable everywhere except possibly on a finite number of smooth surfaces and have normal components which are continuous at these surfaces and (ii) satisfy (2.11) in $\Omega$ and (2.13) on $\partial \Omega$ (but don't necessarily satisfy (2.10)). We show that

$$
W\{\boldsymbol{m}(\boldsymbol{x}), \Omega\}=\inf _{\mathscr{C}} \frac{1}{8 \pi} \int_{\Omega} \boldsymbol{H}^{2}(\boldsymbol{x}) d^{3} \boldsymbol{x}
$$

where the left hand side denotes the magnetostatic energy of the magnetic moment distribution $\boldsymbol{m}(\boldsymbol{x})$ in the region $\Omega$ calculated from Eq. (2.9) and the minimum on the right hand side is over the class $\mathscr{C}$ of vector fields 
$\boldsymbol{H}(\boldsymbol{x})$. To prove (3.2) we define

$$
\delta \boldsymbol{H}(\boldsymbol{x})=\boldsymbol{H}(\boldsymbol{x})-\nabla\left[X_{1}(\boldsymbol{x})+X_{2}(\boldsymbol{x})\right]
$$

where $X_{1}$ and $X_{2}$ are defined in Section II. The boundary conditions (2.13) on $\boldsymbol{H}$ and (2.18) on $X_{1}+X_{2}$ imply that

$$
\delta \boldsymbol{H}_{n}=0 \quad \text { on } \quad \partial \Omega,
$$

while the conditions (2.16), $\nabla^{2} X_{2}=0$ and (2.11) imply

$$
\nabla \cdot(\delta \boldsymbol{H})=0 \quad \text { in } \Omega .
$$

Substituting from (3.3) into the integral to be minimized in (3.2), we obtain

$$
\int_{\Omega} \boldsymbol{H}^{2} d^{3} \boldsymbol{x}=\int_{\Omega}\left\{(\delta \boldsymbol{H})^{2}+\left[\nabla\left(X_{1}+X_{2}\right)\right]^{2}+2 \delta \boldsymbol{H} \cdot \nabla\left(X_{1}+X_{2}\right)\right\} d^{3} \boldsymbol{x} .
$$

The surfaces of discontinuity of $\boldsymbol{H}$ divide $\Omega$ into a finite number of subregions within each of which $\delta \boldsymbol{H}$ is continuous. We apply Green's theorem to the last term of the integrand on the right side of (3.6) separately in each of these subregions of $\Omega$. By using the conditions (3.4) and (3.5) and the continuity of the normal component of $\boldsymbol{H}$ at the surface of discontinuity, we find that the contribution of this term to the integral is zero. Thus (3.6) implies that the integral in (3.2) attains its minimum when $\delta \boldsymbol{H}=0$ and so, by (3.3), equation (3.6) implies that the variational principle in (3.2) is equivalent to the definitions of $W$ and $\boldsymbol{H}$ given in Section II.

Now we may complete the proof of Lemma 1. Let $\boldsymbol{H}^{\prime}$ and $\boldsymbol{H}^{\prime \prime}$ be the fields corresponding to the magnetic moment distributions $\boldsymbol{m}^{\prime}$ and $\boldsymbol{m}^{\prime \prime}$ in the regions $\Omega^{\prime}$ and $\Omega^{\prime \prime}$. They may be calculated by the method of Section II and they satisfy

and

$$
\begin{aligned}
\nabla \cdot \boldsymbol{H}^{\prime}(\boldsymbol{x}) & =-4 \pi \nabla \cdot \boldsymbol{m}^{\prime}(\boldsymbol{x}) \text { for } \quad \boldsymbol{x} \in \Omega^{\prime}, \\
\boldsymbol{H}_{n}^{\prime} & =\boldsymbol{H}_{0, n} \text { on } \partial \Omega^{\prime}, \\
\nabla \cdot \boldsymbol{H}^{\prime \prime}(\boldsymbol{x}) & =-4 \pi \nabla \cdot \boldsymbol{m}^{\prime \prime}(\boldsymbol{x}) \text { for } \quad \boldsymbol{x} \in \Omega^{\prime \prime}
\end{aligned}
$$

$$
\boldsymbol{H}_{n}^{\prime \prime}=\boldsymbol{H}_{0, n} \quad \text { on } \quad \partial \Omega^{\prime \prime} .
$$

The field $\boldsymbol{H}_{1}$ defined by

$$
\boldsymbol{H}_{1}(\boldsymbol{x})= \begin{cases}\boldsymbol{H}^{\prime}(\boldsymbol{x}) & \text { for } \boldsymbol{x} \in \Omega^{\prime} \\ \boldsymbol{H}^{\prime \prime}(\boldsymbol{x}) & \text { for } \boldsymbol{x} \in \Omega^{\prime \prime} \\ \boldsymbol{H}_{0} & \text { for other } \boldsymbol{x}\end{cases}
$$


belongs to the class $\mathscr{C}$. Consequently, by (3.2),

$$
\begin{aligned}
W\{\boldsymbol{m}(\boldsymbol{x}), \Omega\} & \leqq \frac{1}{8 \pi} \int_{\Omega}\left[\boldsymbol{H}_{1}(\boldsymbol{x})\right]^{2} d^{3} \boldsymbol{x} \\
& =\frac{1}{8 \pi}\left\{\int_{\Omega^{\prime}}\left[\boldsymbol{H}^{\prime}(\boldsymbol{x})\right]^{2} d^{3} \boldsymbol{x}+\int_{\Omega^{\prime \prime}}\left[\boldsymbol{H}^{\prime \prime}(\boldsymbol{x})\right]^{2} d^{3} \boldsymbol{x}+\int_{\Omega-\Omega^{\prime}-\Omega^{\prime \prime}} \boldsymbol{H}_{0}^{2} d^{3} \boldsymbol{x}\right\} .
\end{aligned}
$$

So

$$
W\{\boldsymbol{m}(\boldsymbol{x}), \Omega\} \leqq W\left\{\boldsymbol{m}^{\prime}(\boldsymbol{x}), \Omega^{\prime}\right\}+W\left\{\boldsymbol{m}^{\prime \prime}(\boldsymbol{x}), \Omega^{\prime \prime}\right\}+\frac{\boldsymbol{H}_{0}^{2}}{8 \pi}\left\{|\Omega|-\left|\Omega^{\prime}\right|-\left|\Omega^{\prime \prime}\right|\right\}
$$

which is equivalent to (3.1) and the lemma is proved.

\section{The Thermodynamic Limit}

The purpose of this section is to prove the existence of the thermodynamic free energy per unit volume, defined to be $-k T$ times

$$
g\left(\varrho, \boldsymbol{H}_{0}\right) \equiv \lim _{k \rightarrow \infty}\left|\Omega_{k}\right|^{-1} \log Z\left(N_{k}, \Omega_{k}, \boldsymbol{H}_{0}\right) .
$$

Here $Z(N, \Omega, \boldsymbol{H})$ is the partition function for a system of $N$ particles in a region $\Omega$ with the Hamiltonian defined in Section II. We establish the existence of the limit (4.1) for a particular sequence of regions $\Omega_{k}(k=1,2, \ldots)$, all the same shape, which is almost a cube. For all sequences of regions $\Omega_{k}$ which we consider, the corresponding sequences of numbers $N_{k}$ satisfy

$$
\lim _{k \rightarrow \infty}\left[N_{k} /\left|\Omega_{k}\right|\right]=\varrho,
$$

where $0 \leqq \varrho<\varrho_{c}$ and $\varrho_{c}$ is the density of closest packing of the molecules. We extend our existence proof to general sequences of regions $\Omega_{k}$ in the next section. It is possible to extend the proof to allow any finite number of species of molecules, but we do not do this here.

In classical statistical mechanics, the partition function $Z$ is defined by $Z=\left(N ! h^{N d}\right)^{-1} \int \cdots \int \exp (-\beta \mathscr{H}) d \boldsymbol{q}_{1} d \boldsymbol{q}_{2} \ldots d \boldsymbol{q}_{N} d \boldsymbol{p}_{1} d \boldsymbol{p}_{2} \ldots d \boldsymbol{p}_{N}$

where $d$ denotes the number of degrees of freedom of a molecule and $\mathscr{H}$ is the Hamiltonian defined in (2.1). If $N=0$, then we use the definition

$$
Z=\exp \left(\beta \boldsymbol{H}_{0}^{2}|\Omega| / 8 \pi\right) .
$$

To prove the existence of the limit (4.1) we use the method of Ruelle [1] and Fisher [2]. We consider the quantities

$$
g^{*}\left(N, \Omega, \boldsymbol{H}_{0}\right)=\frac{1}{|\Omega|} \log Z^{*}\left(N, \Omega, \boldsymbol{H}_{0}\right)
$$


where

$$
Z^{*}\left(N, \Omega, \boldsymbol{H}_{0}\right) \equiv Z\left(N, \Omega, \boldsymbol{H}_{0}\right) \exp \left(\beta \boldsymbol{H}_{0}^{2}|\Omega| / 8 \pi\right)
$$

with $Z$ defined in (4.3). We call the quantities $g^{*}\left(N, \Omega, \boldsymbol{H}_{0}\right)$ the "modified free energy". First we show that for any sequence of regions $\Omega_{k}$ with an associated sequence of numbers $N_{k}$ satisfying (4.2), the sequence $g^{*}\left(N_{1}, \Omega_{1}, \boldsymbol{H}_{0}\right), g^{*}\left(N_{2}, \Omega_{2}, \boldsymbol{H}_{0}\right), \ldots$ is bounded above.

To obtain this upper bound on $g^{*}$, we replace the non-electromagnetic part of the potential energy by its lower bound

$$
-N u \equiv-N\left(u_{(1)}+u_{(2)}\right)
$$

and replace the electromagnetic potential energy $W$ by its lower bound 0 . The integrals in (4.3) may then be evaluated and we obtain

$$
Z \leqq(N !)^{-1} a^{N} e^{\beta N u}|\Omega|^{N}
$$

where $a^{N}$ is the result of integrating over all the momentum coordinates $\boldsymbol{q}_{1}, \ldots, \boldsymbol{q}_{N}$. Substituting this into (4.5) we find for a sequence of regions $\Omega_{k}$ and a sequence of numbers $N_{k}$,

$$
g^{*}\left(N_{k}, \Omega_{k}, \boldsymbol{H}_{0}\right) \leqq \beta \boldsymbol{H}_{0}^{2} / 8 \pi+\left(N_{k} /\left|\Omega_{k}\right|\right)\left\{\beta u+\log \left(e\left(\left|\Omega_{k}\right| / N_{k}\right) a\right)\right\} .
$$

Now, by (4.2), for any $\varepsilon>0$, for the sequence $\alpha$ of regions $\Omega_{k}$, there is a number $M_{\alpha}(\varepsilon)$ such that

$$
\left|N_{k} /\right| \Omega_{k}|-\varrho|<\varepsilon \quad \text { for } k \geqq M_{\alpha}(\varepsilon) .
$$

Using this inequality with $\varepsilon=\varrho / 2$ in (4.7) we find

$$
g^{*}\left(N_{k}, \Omega_{k}, \boldsymbol{H}_{0}\right) \leqq \frac{\beta \boldsymbol{H}_{0}^{2}}{8 \pi}+\frac{3 \varrho}{2}\left\{\beta u+\log \left(e \frac{2}{\varrho} a\right)\right\}
$$

for $k \geqq M_{\alpha}(\varrho / 2)$. Thus, for all $\varrho>0$, the sequence $g^{*}\left(N_{k}, \Omega_{k}, H_{0}\right)$ is bounded above for all $k$ greater than some finite number. The case $\varrho=0$ requires separate consideration, which will be given later.

All we have to do now to prove the existence of the limit (4.1) for a sequence of domains $\Omega_{k}$ is to prove that the sequences $g^{*}\left(N_{k}, \Omega_{k}, \boldsymbol{H}_{0}\right)$ is nondecreasing. To do this we first prove the inequality

$$
Z^{*}\left(N, \Omega, \boldsymbol{H}_{0}\right) \geqq Z^{*}\left(N^{\prime}, \Omega^{\prime}, \boldsymbol{H}_{0}\right) Z^{*}\left(N^{\prime \prime}, \Omega^{\prime \prime}, \boldsymbol{H}_{0}\right)
$$

where $N=N^{\prime}+N^{\prime \prime}$ and $\Omega^{\prime}$ and $\Omega^{\prime \prime}$ are two disjoint regions both contained in a region $\Omega$ and separated by a distance greater than or equal to $R_{0}$. The regions $\Omega^{\prime}, \Omega^{\prime \prime}$ and $\Omega$ all have surfaces with continuous second derivatives.

The integral in (4.3) is decreased if we confine the region of integration to that part of phase space where $\Omega^{\prime}$ contains $N^{\prime}$ molecules and $\Omega^{\prime \prime}$ contains $N^{\prime \prime}$ molecules so that the rest of $\Omega$ is empty. This restricted 
integral is $N ! /\left(N^{\prime} ! N^{\prime \prime} !\right)$ times the integral in which the first $N^{\prime}$ molecules (labelled $1, \ldots, N^{\prime}$ ) are in $\Omega^{\prime}$ and the other $N^{\prime \prime}$ molecules (labelled $\left.N^{\prime}+1, \ldots, N\right)$ are in $\Omega^{\prime \prime}$.

Thus to prove (4.9) we must show

$$
\exp (-\beta \mathscr{H}) \exp \left(\beta\left[|\Omega|-\left|\Omega^{\prime}\right|-\left|\Omega^{\prime \prime}\right|\right] \boldsymbol{H}_{0}^{2} / 8 \pi\right) \geqq \exp \left(-\beta\left[\mathscr{H}^{\prime}+\mathscr{H}^{\prime \prime}\right]\right)
$$

where $\mathscr{H}^{\prime}$ is the Hamiltonian for those molecules in $\Omega^{\prime}$ with the wall and magnetostatic interactions appropriate to $\partial \Omega^{\prime}$ as boundary and $\mathscr{H}^{\prime \prime}$ similarly for $\Omega^{\prime \prime}$.

For the kinetic energy term in (4.10) we have

$$
K=K^{\prime}+K^{\prime \prime}
$$

so that we can ignore the kinetic energy in proving (4.10). For the two body short range potential energy, the change in going from $\mathscr{H}$ to $\mathscr{H}^{\prime}+\mathscr{H}^{\prime \prime}$ is to drop terms in $\sum_{i, j} U_{i, j}$ for which one molecule is in $\Omega^{\prime}$ and the other in $\Omega^{\prime \prime}$. Since the separation between $\Omega^{\prime}$ and $\Omega^{\prime \prime}$ is greater than or equal to $R_{0}$ these interaction terms are negative by $(2.7)$ and $\mathscr{H}$ is increased by their removal. For the one body potential, the condition (2.8) ensures that the container $\Omega^{\prime}+\Omega^{\prime \prime}$ has a larger potential energy than the enclosing container $\Omega$. So for the whole short range potential energy we obtain

$$
U^{\prime}+U^{\prime \prime} \geqq U \text { in all cases . }
$$

Finally we treat the electromagnetic energy using the lemma of Section III which shows that

$$
W-\frac{1}{8 \pi} \boldsymbol{H}_{0}^{2}\left(|\Omega|-\left|\Omega^{\prime}\right|-\left|\Omega^{\prime \prime}\right|\right) \leqq W^{\prime}+W^{\prime \prime} .
$$

This completes the proof of (4.10) and thus of (4.9).

We now introduce the "standard sequences" $\Omega_{s, k}$ and $N_{s, k}(k=1,2, \ldots)$ to prove the existence of the limit (4.1). The sequences are

where

$$
\begin{aligned}
& \Omega_{s, k}=\text { a cube with "smooth edges" of side } 2^{k} \xi-R_{0} \\
& N_{s, k}=8^{k} v
\end{aligned}
$$

$$
\xi \equiv(v / \varrho)^{1 / 3}
$$

and $v$ is a positive integer chosen so that $\xi>R_{0}$. We define

$$
G_{k}\left(\varrho, \boldsymbol{H}_{0}\right) \equiv g^{*}\left(N_{s, k}, \Omega_{s, k}, \boldsymbol{H}_{0}\right) .
$$

We recall that in Sections II and III we proved results for regions with surfaces which have continuous second derivatives. Unfortunately this does not hold for cubes. In order to have a sequence of regions which do have smooth enough surfaces, we construct "cubes with 'smooth edges". 


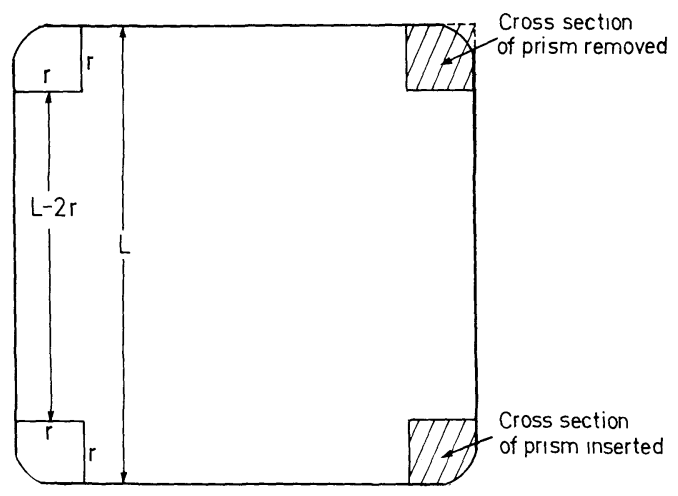

Fig. 1. A cross section of a cube with "smooth edges"

To construct a cube with "smooth edges" we take first an ordinary cube of side, say, $L$. From each corner of this cube we remove a small cube of side $r$ where $r \ll L$. From each edge of length $L-2 r$ of the remaining object we remove a prism of length $L-2 r$ and square base of side $r$. In place of the cubes removed from the corners we place objects best described as made up of the points which form the solution set of the inequalities

$$
x \geqq 0, \quad y \geqq 0, \quad z \geqq 0 \quad \text { and } \quad x^{4}+y^{4}+z^{4} \leqq r^{4}
$$

where $x, y$ and $z$ are rectangular coordinates. In place of the prisms removed from the edges we place prisms of length $L-2 r$ and cross section those points making up the solution set of the inequalities

$$
x \geqq 0, \quad y \geqq 0 \quad \text { and } \quad x^{4}+y^{4} \leqq r^{4},
$$

where $x$ and $y$ are rectangular coordinates. A sketch of a cross section of a cube with smooth edges is shown in Fig. 1.

If we call the difference in volume between an ordinary cube of side $L$ and a cube with smooth edges of side $L, V_{L}$, then we have

$$
V_{L} \leqq 12 L r^{2} \text {, }
$$

so that for small $r$, the volume of the smoothed cube is not very different from that of the unsmoothed cube. It should be noted that the surface of a cube with smooth edges indeed has a continuous second derivative.

From (4.9) it follows by a natural generalization that

$$
Z^{*}\left(N, \Omega, \boldsymbol{H}_{0}\right) \geqq \prod_{i=1}^{8} Z^{*}\left(N^{i}, \Omega^{i}, \boldsymbol{H}_{0}\right)
$$


where $N=\sum_{i=1}^{8} N^{i}$ and $\Omega^{1}, \ldots, \Omega^{8}$ are non-overlapping regions contained in $\Omega$. We take $\Omega=\Omega_{s, k+1}$ of the sequence (4.13) and $\Omega^{1}, \ldots, \Omega^{8}$ to be eight cubes with smooth edges and side $2^{k} \xi \rightarrow R_{0}$ separated by corridors of width $R_{0}$ and tucked into the corners of $\Omega$, where the parameter $r$ describing the smooth corners of $\Omega$ and all the $\Omega^{i}$ is the same in all cases. Then we have, since $\varrho<\varrho_{c}$,

$$
8^{-k-1} \log Z^{*}\left(N, \Omega, \boldsymbol{H}_{0}\right) \geqq \sum_{i=1}^{8} 8^{-k-1} \log Z^{*}\left(N^{i}, \Omega^{i}, \boldsymbol{H}_{0}\right)
$$

or if $N=8^{k+1} v, N^{i}=8^{k} v$ and $\Omega=\Omega_{s, k+1}$,

$$
8^{-k-1} \log Z^{*}\left(N, \Omega_{s, k+1}, \boldsymbol{H}_{0}\right) \geqq 8^{-k} \log Z^{*}\left(N / 8, \Omega_{s, k}, \boldsymbol{H}_{0}\right)
$$

so that the sequence $G_{k}\left(\varrho, H_{0}\right)$ is non-decreasing and, because of (4.18) and (4.8) the limit (4.1) exists for the sequence of regions described in (4.13).

Finally we consider the case $\varrho=0$. We extend the set of standard sequences to include

$$
\begin{aligned}
& \Omega_{s, k}=\text { a cube with smooth edges of side } 2^{k} \xi-R_{0} \\
& N_{s, k}=0
\end{aligned}
$$

for which $\lim _{k \rightarrow \infty} N_{s, k} /\left|\Omega_{s, k}\right|=0$. By (4.3b) we have

so that

$$
Z\left(0, \Omega_{s, k}, \boldsymbol{H}_{0}\right)=\exp \left(-\beta \boldsymbol{H}_{0}^{2}\left|\Omega_{s, k}\right| / 8 \pi\right)
$$

and

$$
Z *\left(0, \Omega_{s, k}, \boldsymbol{H}_{0}\right)=1
$$

$$
G_{k}\left(0, \boldsymbol{H}_{0}\right)=0 \text { for all } k \text {. }
$$

Thus $\lim _{k \rightarrow \infty} G_{k}\left(0, \boldsymbol{H}_{0}\right)$ exists, so that the limit (4.1) exists for $0 \leqq \varrho<\varrho_{c}$.

\section{General Sequences of Regions}

We follow the technique of Fisher [2] to prove that the free energy defined by (4.1) exists for more general sequences of regions $\left\{\Omega_{j}\right\}$ and is the same as that for the standard sequence of regions defined in (4.13). Firstly we prove that, for suitable sequences $\left\{\Omega_{j}\right\}$

where

$$
\begin{gathered}
\lim _{j \rightarrow \infty} \inf g^{*}\left(N_{j}, \Omega_{j}, \boldsymbol{H}_{0}\right) \geqq G\left(\varrho, \boldsymbol{H}_{0}\right) \\
G\left(\varrho, \boldsymbol{H}_{0}\right) \equiv \lim _{k \rightarrow \infty} G_{k}\left(\varrho, \boldsymbol{H}_{0}\right)
\end{gathered}
$$


is the modified free energy density given by the standard sequence (4.13) and

$$
g^{*}\left(N_{j}, \Omega_{j}, \boldsymbol{H}_{0}\right)=\frac{1}{\left|\Omega_{j}\right|} \log Z^{*}\left(N_{j}, \Omega_{j}, \boldsymbol{H}_{0}\right)
$$

for a sequence $\left\{N_{j}\right\}$ such that $\lim _{j \rightarrow \infty} N_{j} /\left|\Omega_{j}\right|=\varrho$. Then we prove

$$
\lim _{j \rightarrow \infty} \sup g^{*}\left(N_{j}, \Omega_{j}, \boldsymbol{H}_{0}\right) \leqq G\left(\varrho, \boldsymbol{H}_{0}\right) .
$$

Taken together, (5.1) and (5.3) imply

$$
\lim _{j \rightarrow \infty} g^{*}\left(N_{j}, \Omega_{j}, \boldsymbol{H}_{0}\right)=G\left(\varrho, \boldsymbol{H}_{0}\right) .
$$

To prove (5.1) we consider a maximal filling of the region $\Omega_{j}$ by $n_{j, k}$ regions $\Omega_{s, k}$ of the standard sequence (4.13), the cubes $\Omega_{s, k}$ being kept a distance $R_{0}$ apart in this process. The first condition we impose on the sequence of regions $\left\{\Omega_{j}\right\}$ is that the surfaces of all the regions have continuous second derivatives, otherwise we cannot define the Hamiltonian as in Section II. We then apply the inequality (4.9) with the same density $\varrho^{\prime}$ in each cube. This gives

$$
Z^{*}\left(N_{j, k}, \Omega_{j}, \boldsymbol{H}_{0}\right) \geqq \prod_{r=1}^{n_{\jmath, k}} Z^{*}\left(N_{s, k}, \Omega_{s, k}, \boldsymbol{H}_{0}\right)
$$

where $N_{j, k}=N_{s, k} n_{j, k}$. The corresponding inequality for the modified free energy is then

$$
g^{*}\left(N_{j, k}, \Omega_{j}, \boldsymbol{H}_{0}\right) \geqq \frac{n_{j, k}\left|\Omega_{s, k}\right|}{\left|\Omega_{j}\right|} G_{k}\left(\varrho, \boldsymbol{H}_{0}\right) .
$$

Thus we have

$$
\lim _{j \rightarrow \infty} \inf g^{*}\left(N_{j . k}, \Omega_{j}, H_{0}\right) \geqq \lim _{j \rightarrow \infty} \inf \frac{n_{j, k}\left|\Omega_{s, k}\right|}{\left|\Omega_{j}\right|} G_{k}\left(\varrho, H_{0}\right)
$$

for all $k$. Thus (5.1) will hold if

$$
\lim _{k \rightarrow \infty} \lim _{j \rightarrow \infty} \frac{n_{j, k}\left|\Omega_{s, k}\right|}{\left|\Omega_{j}\right|}=1 .
$$

To establish (5.3) we enclose $\Omega_{j}$ in the smallest possible region of the standard sequence (4.13), $\Omega_{s, K(j)}$, which keeps the distance of $\Omega_{j}$ from the boundary of $\Omega_{s, K(j)}$ greater than or equal to $R_{0}$. We then construct a maximal filling of the volume between $\Omega_{j}$ and $\Omega_{s, K(j)}$ by $m_{j, k}$ smaller standard regions $\Omega_{s, k}$ with a spacing of at least $R_{0}$. The inequality (4.9) applied to the subdivision of $\Omega_{s, K(j)}$ gives

$$
g^{*}\left(N_{s, K}^{\prime}, \Omega_{s, K}, \boldsymbol{H}_{0}\right) \geqq \frac{\left|\Omega_{j}\right|}{\left|\Omega_{s, K}\right|} g^{*}\left(N_{j, k}, \Omega_{j}, \boldsymbol{H}_{0}\right)+m_{j, k} \frac{\left|\Omega_{s, k}\right|}{\left|\Omega_{s, K}\right|} g^{*}\left(N_{s, k}, \Omega_{s, k}, \boldsymbol{H}_{0}\right)
$$


where $N_{s, K}^{\prime}=N_{j, k}+m_{j, k} N_{s, k}$. Manipulating (5.8) we obtain

$$
\begin{aligned}
g^{*}\left(N_{j, k}, \Omega_{j}, \boldsymbol{H}_{0}\right) \leqq & \left\{\frac{\left|\Omega_{s, K}\right|}{\left|\Omega_{j}\right|}-m_{j, k} \frac{\left|\Omega_{s, k}\right|}{\left|\Omega_{j}\right|}\right\} g^{*}\left(N_{s, K}^{\prime}, \Omega_{s, K}, \boldsymbol{H}_{0}\right) \\
& +m_{j, k} \frac{\left|\Omega_{s, k}\right|}{\left|\Omega_{j}\right|}\left\{g^{*}\left(N_{s, K}^{\prime}, \Omega_{s, K}, \boldsymbol{H}_{0}\right)-g^{*}\left(N_{s, k}, \Omega_{s, k}, \boldsymbol{H}_{0}\right)\right\} .
\end{aligned}
$$

If we have the two conditions

$$
\lim _{k \rightarrow \infty} \lim _{j \rightarrow \infty}\left|\Omega_{j}\right|^{-1}\left\{\left|\Omega_{s, K}\right|-m_{j, k}\left|\Omega_{s, k}\right|\right\}=1
$$

and there exists $A$ such that

$$
m_{j, k} \frac{\left|\Omega_{s, k}\right|}{\left|\Omega_{j}\right|} \leqq A \quad \text { for all } j \text { and } k,
$$

then $\lim _{k \rightarrow \infty} \lim _{j \rightarrow \infty} \frac{N_{s, K}^{\prime}}{\left|\Omega_{s, K}\right|}=\varrho$ and by the continuity of $G\left(\varrho, \boldsymbol{H}_{0}\right)$ in $\varrho$ which we prove in the next section we have

$$
\lim _{j \rightarrow \infty} \sup g^{*}\left(N_{j, k}, \Omega_{j}, \boldsymbol{H}_{0}\right) \leqq G\left(\varrho, \boldsymbol{H}_{0}\right) .
$$

We have proved (5.1) and (5.3) and thus (5.4) for all sequences of regions satisfying (5.7) and (5.9a), (5.9b). A discussion of conditions on the sequences of regions $\left\{\Omega_{j}\right\}$ which yield (5.7) and (5.9a), (5.9b) may be found in Fisher [2].

For systems with electromagnetic interactions, it is important to be able to describe "shape-dependent" effects in the thermodynamic behaviour. Unfortunately, it is not immediately clear what we mean by the "shape" of an infinitely large region. To overcome this difficulty we consider sequences of regions all of the same shape. As the first member of the sequence we take a finite region $\omega_{1}$ with a surface with continuous second derivative and with a non-zero Peano-Jordan content [9]. We construct the $n^{\text {th }}$ member of the sequence $\left\{\omega_{n}\right\}$ from $\omega_{1}$ by multiplying every linear dimension of $\omega_{1}$ by $n$. We sketch this construction for $\omega_{3}$ in Fig. 2. The volume of $\omega_{n}$ is then

$$
\left|\omega_{n}\right|=n^{3}\left|\omega_{1}\right| .
$$

The free energy of the sequence $\left\{\omega_{n}\right\}$ is then the free energy for a given shape, namely that of $\omega_{1}$. If the sequence $\left\{\omega_{n}\right\}$ obeys (5.7) and (5.9a), (5.9 b), then the free energy for the shape $\omega_{1}$ is equal to

$$
-k T\left\{G\left(\varrho, \boldsymbol{H}_{0}\right)-\beta \boldsymbol{H}_{0}^{2} / 8 \pi\right\} .
$$

In addition the free energy does not depend upon the shape $\omega_{1}$. We now show that the condition that $\omega_{1}$ have a non-zero Peano-Jordan content 


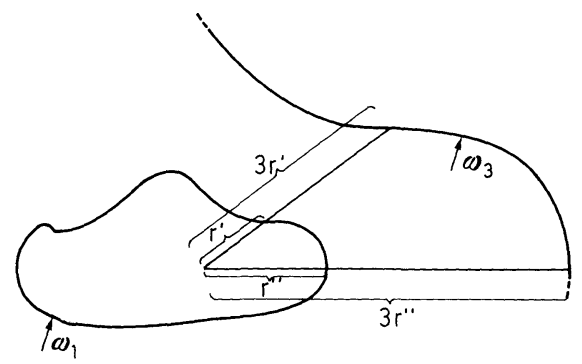

Fig. 2. Sketch of construction of $\omega_{3}$ from $\omega_{1}$

is sufficient for (5.7), (5.9a) and (5.9 b) to be satisfied and so for the modified free energy of the sequence $\left\{\omega_{n}\right\}$ to be $G\left(\varrho, \boldsymbol{H}_{0}\right)$.

If we construct a maximal filling of $\omega_{q}$ by cubes of volume $8^{k} \xi^{3}$, then we are, at the same time, constructing a maximal filling of $\omega_{1}$ by cubes of volume $8^{k} \xi^{3} / q^{3}$ and so we can say $n_{q, k} \leqq q^{3}\left|\omega_{1}\right| /\left(8^{k} \xi^{3}\right)$. Now

Thus

$$
\left(2^{k} \xi-R_{0}\right)^{3} \geqq\left|\Omega_{s, k}\right| \geqq\left(2^{k} \xi-R_{0}-2 r\right)^{3} .
$$

so that

$$
\frac{n_{q, k} 8^{k} \xi^{3}}{q^{3}\left|\omega_{q}\right|} \geqq \frac{n_{q, k}\left|\Omega_{s, k}\right|}{\left|\omega_{q}\right|} \geqq \frac{n_{q, k} 8^{k} \xi^{3}}{q^{3}\left|\omega_{1}\right|}\left\{1-\frac{\left(R_{0}+2 r\right)}{2^{k} \xi}\right\}^{3}
$$

$$
\frac{n_{q, k} 8^{k} \xi^{3} / q^{3}}{\left|\omega_{1}\right|} \geqq \frac{n_{q, k}\left|\Omega_{s, k}\right|}{\left|\omega_{q}\right|} \geqq \frac{n_{q, k} k^{k} \xi^{3} / q^{3}}{\left|\omega_{1}\right|}\left\{1-\frac{\left(R_{0}+2 r\right)}{2^{k} \xi}\right\}^{3} .
$$

Now $n_{q, k} 8^{k} \xi^{3} / q^{3}$ is the volume of an approximation to the region $\omega_{1}$ by cubes of side $2^{k} \xi / q$. Since $\omega_{1}$ has a non-zero Peano-Jordan content, $n_{q, k} 8^{k} \xi^{3} /\left(q^{3}\left|\omega_{1}\right|\right) \rightarrow 1$ as $q \rightarrow \infty$ if $2^{k} \xi / q \rightarrow 0$. But to prove (5.7) from (5.12) we require $3 R_{0} /\left(2^{k} \xi\right) \rightarrow 0$ also. So if we take

$$
k=\left[\left(\frac{1}{2} \log q-\log \xi\right) /(\log 2)\right]
$$

where $[x]$ is the largest integer smaller than $x$, then $2^{k} \xi \sim \sqrt{q}$. Thus (5.7) holds as we take the limit $q \rightarrow \infty$ with $k$ as in (5.13).

We prove $(5.9$ b) by

$$
0<\frac{m_{q, k}\left|\Omega_{s, k}\right|}{\left|\omega_{q}\right|}<\frac{8^{K(q)} \xi^{3}}{\left|\omega_{q}\right|} \leqq \frac{8 q^{3}\left|\Omega_{s, K(1)}\right|}{q^{3}\left|\omega_{1}\right|}=\frac{8\left|\Omega_{s, K(1)}\right|}{\left|\omega_{1}\right|}
$$

which proves (5.9b) since both $\Omega_{s, K(1)}$ and $\omega_{1}$ have finite non-zero PeanoJordan content.

To prove (5.9a) we use (5.11) to write

$$
\frac{1}{\left|\omega_{q}\right|}\left\{\left|\Omega_{s, K(q)}\right|-m_{q, k}\left|\Omega_{s, k}\right|\right\}=\frac{1}{\left|\omega_{1}\right|}\left\{\frac{8^{K(q)} \xi^{3}}{q^{3}}-m_{q, k} \frac{8^{k} \xi^{3}}{q^{3}}\right\}\left[1-0\left(\frac{3\left(R_{0}+2 r\right)}{2^{k} \xi}\right)\right] \text {. }
$$


The two terms in the first bracket on the right hand side of (5.15) are the volume of the best exterior approximation to $\omega_{1}$ by cubes of side $2^{k} \xi / q$. We take the limit $q \rightarrow \infty$ with $k$ as in (5.13). Because $\omega_{1}$ has a PeanoJordan content and because by (5.13) $2^{k} \xi / q \rightarrow 0$,

$$
\lim _{q \rightarrow \infty} \frac{1}{\left|\omega_{q}\right|}\left\{\left|\Omega_{s, K(q)}\right|-m_{q, k}\left|\Omega_{s, k}\right|\right\}=1 .
$$

Thus (5.9a) is established, so it is sufficient, for the modified free energy of the sequence $\left\{\omega_{n}\right\}$ to exist and equal $G\left(\varrho, \boldsymbol{H}_{0}\right)$, that $\omega_{1}$ have a finite non-zero Peano-Jordan content.

It may be noted that it is possible to extend the definition (4.1) of the free energy to include of regions $\left\{\Omega_{j}\right\}$ which do not have a Peano-Jordan content. However, it would be necessary either for there to be an alternative definition of volume for the regions or for the volume of both the exterior and interior approximations to the regions (that is the outer content and the inner content) to differ from one another by an amount which became negligible with respect to the inner content as $j \rightarrow \infty$. This extension would not be possible with the shaped sequences introduced in this section as the ratio of the difference to the inner content would remain constant in the sequence $\left\{\omega_{n}\right\}$.

The free energy (4.1) for the sequences of regions with shape introduced in this section is shape independent because the regions obey (5.7), (5.9a) and $(5.9 \mathrm{~b})$. In particular, if the Hamiltonian $\mathscr{H}$ introduced in Section II is rotationally invariant, then for a given magnetic field $\boldsymbol{H}_{0}$, the free energy for a shape is independent of the orientation of the shape with respect to the magnetic field. That is, the free energy depends only on the magnitude of the magnetic field imposed at the boundary of the container. This is not generally true for any Hamiltonian, since the effect of rotating the system with respect to the magnetic field may be to change the Hamiltonian for the system we are considering. If the Hamiltonian is changed then we may reasonably expect the free energy of the system to change.

The physical reason for the shape independence of the free energy is that the system can form magnetic domains. The domains will arrange themselves so that the boundary conditions (2.13) are satisfied. The shape independence of the free energy density then follows because the domains all have the same free energy density, and the contribution to the free energy density of the whole system from the boundaries between the magnetic domains is negligible.

\section{Simple Properties of the Free Energy}

We first establish that the modified free energy $G\left(\varrho, \boldsymbol{H}_{0}\right)$ is a concave function of $\varrho$ on $0 \leqq \varrho<\varrho_{c}$. We apply the inequality (4.9) to four regions 
$\Omega_{s, k}$ with density $\varrho^{\prime}$ placed in the upper half of a region $\Omega_{s, k+1}$, and four regions with a density $\varrho^{\prime \prime}$ placed in the lower half of $\Omega_{s, k+1}$. The inequality for the free energy implied by Eq. (4.18) is then

$$
G_{k+1}\left(\varrho, \boldsymbol{H}_{0}\right) \geqq \frac{4\left|\Omega_{s, k}\right|}{\left|\Omega_{s, k+1}\right|}\left[G_{k}\left(\varrho^{\prime \prime}, \boldsymbol{H}_{0}\right)+G_{k}\left(\varrho^{\prime}, \boldsymbol{H}_{0}\right)\right]
$$

where $\varrho=\varrho^{\prime} / 2+\varrho^{\prime \prime} / 2$. Letting $k \rightarrow \infty$ in Eq. (6.1) we obtain

$$
G\left(\varrho^{\prime} / 2+\varrho^{\prime \prime} / 2, \boldsymbol{H}_{0}\right) \geqq \frac{1}{2} G\left(\varrho^{\prime}, \boldsymbol{H}_{0}\right)+\frac{1}{2} G\left(\varrho^{\prime \prime}, \boldsymbol{H}_{0}\right) .
$$

The modified free energy $G\left(\varrho, \boldsymbol{H}_{0}\right)$ is thus concave on $0 \leqq \varrho<\varrho_{c}$ and the free energy $-k T g\left(\varrho, \boldsymbol{H}_{0}\right)$ of $(4.1)$ is thus convex on $0 \leqq \varrho<\varrho_{c}$. This means that both functions are continuous in $\varrho$ on $0<\varrho<\varrho_{c}$ since by Eq. (4.8) they are both bounded ([10], Theorem 111). We required this property in the last section to establish the existence of the limit (4.1) for general sequences of regions and its independence of the particular sequences of regions used. From Eq. (6.2) we may deduce the more general convexity property

$$
g\left(\sum_{i} p_{i} \varrho_{i}, \boldsymbol{H}_{0}\right) \geqq \sum_{i} p_{i} g\left(\varrho_{i}, \boldsymbol{H}_{0}\right)
$$

where $\sum_{i} p_{i}=1[10]$.

Next we show that the free energy density is convex in $\boldsymbol{H}_{0}$, the external field applied at the boundary. That is we prove

where

$$
g\left(\varrho, \boldsymbol{H}_{0}\right) \geqq \frac{1}{2} g\left(\varrho, \boldsymbol{H}_{1}\right)+\frac{1}{2} g\left(\varrho, \boldsymbol{H}_{2}\right)
$$

$$
2 \boldsymbol{H}_{0}=\boldsymbol{H}_{1}+\boldsymbol{H}_{2} \text {. }
$$

We define $x, y$ and $z$ directions with $x$ along the vector $\boldsymbol{H}_{1}-\boldsymbol{H}_{2}$ and $z$ at right angles to $\boldsymbol{H}_{1}$ and $\boldsymbol{H}_{2}$ so that $\boldsymbol{H}_{1}$ and $\boldsymbol{H}_{2}$ are both in the $x-y$ plane. We consider a set of $2 M$ cuboidal regions of dimensions $L \times d \times L$ with edges smoothed in the same way as those of the standard sequence (4.13). We lay them in a stack of size $L \times\left(2 M d+(2 M-1) R_{0}\right) \times L$ with a separation $R_{0}$ between each region. We then enclose the stack of regions in another region of dimensions $(L+6 D) \times\left(2 M\left(d+R_{0}\right)\right) \times L$ which overlaps each end of the stack of regions by a distance $3 D$ and is distant $R_{0} / 2$ from the $x-z$ faces of the stack of regions. We illustrate this array of regions in Fig. 3. We call the thin regions $\Omega_{L, d}$ and number them from 1 to $2 M$ as in Fig. 3 and call the enclosing region $\Omega_{L}$. We choose the number of regions in the stack so that

$$
2 M \leqq L /\left(d+R_{0}\right)<2(M+1) .
$$




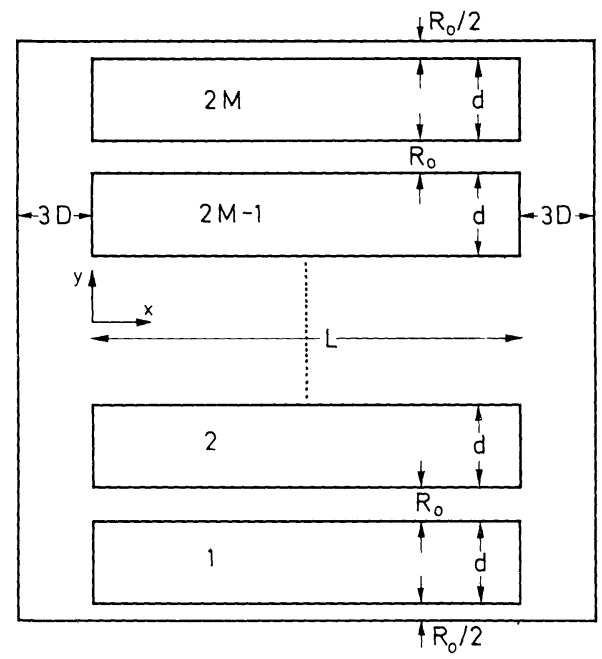

Fig. 3. Sketch of $2 M$ regions $\Omega_{L, d}$ enclosed within the region $\Omega_{L}$

To prove the inequality (6.4) we bound the partition function $Z\left(N, \Omega_{L}, \boldsymbol{H}_{0}\right)$ below by an integral over those parts of phase space in which the $N$ particles lie in the regions $\Omega_{L, d}$ with an equal density in each. We can obtain an upper bound on the electromagnetic potential energy of this restricted system from the minimization principle of Section III. In fact we arrange that on the surfaces of the odd numbered regions $\Omega_{L, d}$ the trial magnetic field $\boldsymbol{H}^{\prime}$ should have a normal component equal to that of $\boldsymbol{H}_{1}$ while on the surface of the even numbered regions the magnetic field has a normal component equal to that of $\boldsymbol{H}_{2}$. Within the regions $\Omega_{L, d}$ we allow $\boldsymbol{H}^{\prime}$ to satisfy $\boldsymbol{\nabla} \times \boldsymbol{H}^{\prime}=0$. The field outside these regions is defined below in Eq. (6.9). This enables the phase space integrations for the bound on the partition function to be performed and we obtain

$Z\left(N, \Omega_{L}, \boldsymbol{H}_{0}\right) \geqq\left(Z\left(N / 2 M, \Omega_{L, d}, \boldsymbol{H}_{1}\right)\right)^{M}\left(Z\left(N / 2 M, \Omega_{L, d}, \boldsymbol{H}_{2}\right)\right)^{M} \exp \left[-\beta W\left(\boldsymbol{H}^{\prime}\right)\right]$,

where $W\left(\boldsymbol{H}^{\prime}\right)$ is the potential energy of a field $\boldsymbol{H}^{\prime}$ in those parts of $\Omega_{L}$ not occupied by regions $\Omega_{L, d}$. $\boldsymbol{H}^{\prime}$ is a field belonging to the class $\mathscr{C}$ and for which

$\boldsymbol{H}_{n}^{\prime}=\boldsymbol{H}_{0, n} \quad$ on the surface of $\Omega_{L}$ and

$\boldsymbol{H}_{n}^{\prime}=\boldsymbol{H}_{1, n} \quad$ on the surfaces of the odd numbered regions $\Omega_{L, d}$

$\boldsymbol{H}_{n}^{\prime}=\boldsymbol{H}_{2, n} \quad$ on the surfaces of the even numbered regions $\Omega_{L, d}$.

In Fig. 4 we illustrate the left hand end (cf. Fig. 3) of the regions $\Omega_{L, d}$ and the adjacent part of the surface of $\Omega_{L}$. 


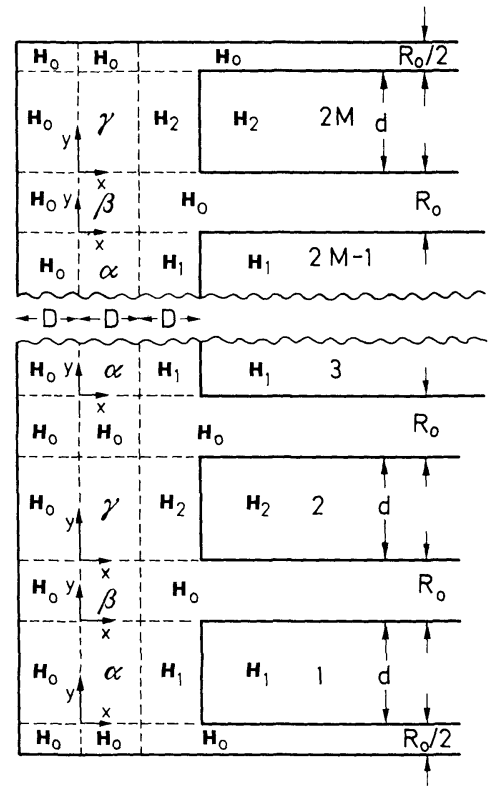

Fig. 4. Left-hand ends of regions $\Omega_{L, d}$ and adjacent part of boundary of $\Omega_{L}$ showing values of the magnetic field $\boldsymbol{H}^{\prime}$

For the field $\boldsymbol{H}^{\prime}$ we choose $\boldsymbol{H}_{z}^{\prime}=0$ everywhere and the $x$ and $y$ components as shown in Fig. 4 with the fields $\boldsymbol{H}_{\alpha}, \boldsymbol{H}_{\beta}$ and $\boldsymbol{H}_{\gamma}$ defined by

$$
\begin{aligned}
\boldsymbol{H}_{\alpha, x} & =\boldsymbol{H}_{0, x}+\left(\boldsymbol{H}_{1, x}-\boldsymbol{H}_{0, x}\right) x / D \\
\boldsymbol{H}_{\alpha, y} & =\boldsymbol{H}_{0, y}-\left(\boldsymbol{H}_{1, x}-\boldsymbol{H}_{0, x}\right) y / D \\
\boldsymbol{H}_{\beta, x} & =\boldsymbol{H}_{0, x} \\
\boldsymbol{H}_{\beta, x} & =\boldsymbol{H}_{0, y}-\left(\boldsymbol{H}_{1, x}-\boldsymbol{H}_{0, x}\right) d / D \\
H_{\gamma, x} & =\boldsymbol{H}_{0, x}+\left(\boldsymbol{H}_{2, x}-\boldsymbol{H}_{0, x}\right) x / D \\
H_{\gamma, y} & =\boldsymbol{H}_{0, y}-\left(\boldsymbol{H}_{1, x}-\boldsymbol{H}_{0, x}\right) d / D-\left(\boldsymbol{H}_{2, x}-\boldsymbol{H}_{0, x}\right) y / D .
\end{aligned}
$$

Note that each of the regions $\alpha, \beta$ and $\gamma$ has been given, for the purposes of describing this field, its own set of $x-y$ axes.

At the right hand end of the stack of regions we make the field $\boldsymbol{H}^{\prime}$ change back to $\boldsymbol{H}_{0}$ in exactly the same way as we changed it to $\boldsymbol{H}_{1}$ or $\boldsymbol{H}_{2}$ at the left hand end. Within the regions $\Omega_{L, d}$ of course, the field is affected by the various configurations taken by the molecules within the regions so that we can obtain the inequality (6.7). The field $\boldsymbol{H}^{\prime}$ is thus continuous and differentiable everywhere within $\Omega_{L}$ except on a finite number of surfaces. On these surfaces the normal component of $\boldsymbol{H}^{\prime}$ is continuous. The field $\boldsymbol{H}^{\prime}$ obeys Eq. (2.11) within $\Omega_{L}$ and Eq. (2.13) on 
the surface of $\Omega_{L}$. Thus $\boldsymbol{H}^{\prime} \in \mathscr{C}$, and we may use it in Eq. (6.7). An exact calculation gives

$$
\begin{aligned}
W\left(\boldsymbol{H}^{\prime}\right)= & M d L D /(4 \pi)\left\{\left(\boldsymbol{H}_{1, x}-\boldsymbol{H}_{0, x}\right)^{2}\left(\frac{2}{3}+\frac{R_{0} d}{D^{2}}+\frac{2 d^{2}}{3 D^{2}}\right)\right. \\
& \left.-2 \boldsymbol{H}_{0, y} \cdot\left(\boldsymbol{H}_{1, x}-\boldsymbol{H}_{0, x}\right)\left(\frac{d+R_{0}}{D}\right)+\boldsymbol{H}_{1}^{2}+\boldsymbol{H}_{2}^{2}-2 \boldsymbol{H}_{0}^{2}\right\}+W\left(\boldsymbol{H}_{0}\right) .
\end{aligned}
$$

Substituting this result in (6.7) and taking the logarithm of both sides, we obtain

$$
\begin{aligned}
g\left(\varrho, \Omega_{L}, \boldsymbol{H}_{0}\right) \geqq \frac{M\left|\Omega_{L, d}\right|}{\left|\Omega_{L}\right|}\left\{g\left(\varrho^{\prime}, \Omega_{L, d}, \boldsymbol{H}_{1}\right)+g\left(\varrho^{\prime}, \Omega_{L, d}, \boldsymbol{H}_{2}\right)\right\} \\
-\frac{\beta}{\left|\Omega_{L}\right|}\left[\frac { M d L D } { 4 \pi } \left\{\left(\boldsymbol{H}_{1, x}-\boldsymbol{H}_{0, x}\right)^{2}\left(\frac{2}{3}+\frac{R_{0} d}{D^{2}}+\frac{2 d^{2}}{3 D^{2}}\right)\right.\right. \\
\left.\left.\quad-2 \boldsymbol{H}_{0, y} \cdot\left(\boldsymbol{H}_{1, x}-\boldsymbol{H}_{0, x}\right)\left(\frac{d+R_{0}}{D}\right)+\boldsymbol{H}_{1}^{2}+\boldsymbol{H}_{2}^{2}-2 \boldsymbol{H}_{0}^{2}\right\}+W\left(\boldsymbol{H}_{0}\right)\right]
\end{aligned}
$$

where

and

$$
\varrho^{\prime}=\frac{\left|\Omega_{L}\right|}{2 M\left|\Omega_{L, d}\right|} \varrho
$$

$$
g\left(\varrho, \Omega, \boldsymbol{H}_{0}\right) \equiv \frac{1}{|\Omega|} \log Z\left(N, \Omega, \boldsymbol{H}_{0}\right) .
$$

We now take $d=L^{1 / 3}$ so that $M \sim L^{2 / 3}$ and let $L \rightarrow \infty$. We then obtain

$g\left(\varrho, \boldsymbol{H}_{0}\right) \geqq \frac{1}{2} \lim _{L \rightarrow \infty} \sup g\left(\varrho^{\prime}, \Omega_{L, L^{1 / 3}}, \boldsymbol{H}_{1}\right)+\frac{1}{2} \lim _{L \rightarrow \infty} \sup g\left(\varrho^{\prime}, \Omega_{L, L^{1 / 3}}, \boldsymbol{H}_{2}\right)$.

Unfortunately the sequence of cuboidal regions of size $L \times L^{1 / 3} \times L$ do not obey (5.9b) as $L \rightarrow \infty$ so (6.13) does not immediately give the result (6.4). But this sequence of regions does obey condition (5.7) so that Eq. (5.1) holds. This means that we may deduce

$$
\lim _{L \rightarrow \infty} \inf g\left(\varrho^{\prime}, \Omega_{L, L^{1 / 3}}, \boldsymbol{H}_{0}\right) \geqq g\left(\varrho, \boldsymbol{H}_{0}\right) .
$$

If we now put $\boldsymbol{H}_{1}=\boldsymbol{H}_{2}=\boldsymbol{H}_{0}$ in Eq. (6.13) we obtain

$$
\lim _{L \rightarrow \infty} \sup g\left(\varrho^{\prime}, \Omega_{L, L^{1 / 3}}, \boldsymbol{H}_{0}\right) \leqq g\left(\varrho, \boldsymbol{H}_{0}\right) .
$$

From (6.14) and (6.15) we deduce

$$
\lim _{L \rightarrow \infty} \sup g\left(\varrho^{\prime}, \Omega_{L, L^{1 / 3}}, \boldsymbol{H}_{0}\right)=g\left(\varrho, \boldsymbol{H}_{0}\right) .
$$


Substituting (6.16) into (6.13) we obtain

$$
g\left(\varrho, \boldsymbol{H}_{1} / 2+\boldsymbol{H}_{2} / 2\right) \geqq \frac{1}{2} g\left(\varrho, \boldsymbol{H}_{1}\right)+\frac{1}{2} g\left(\varrho, \boldsymbol{H}_{2}\right),
$$

the result we wanted to establish.

We have shown that the free energy exists and that

$$
f\left(\varrho, \boldsymbol{H}_{0}\right) \equiv-k T g\left(\varrho, \boldsymbol{H}_{0}\right)=-k T\left(G\left(\varrho, \boldsymbol{H}_{0}\right)-\beta \boldsymbol{H}_{0}^{2} / 8 \pi\right)
$$

and thus that the free energy $f\left(\varrho, \boldsymbol{H}_{0}\right)$ is a convex function of $\varrho$ on $0 \leqq \varrho<\varrho_{c}$ and of $\boldsymbol{H}_{0}$. From these properties we can deduce the following properties of $f\left(\varrho, \boldsymbol{H}_{0}\right)$.

a) Continuity. $f\left(\varrho, \boldsymbol{H}_{0}\right)$ is continuous in $\varrho$ on $0<\varrho<\varrho_{c}$ and in $\boldsymbol{H}_{0, x}$, $\boldsymbol{H}_{0, y}$ and $\boldsymbol{H}_{0, z}$.

b) Differentiability. The right and left hand derivatives

and

$$
\begin{aligned}
& \frac{\partial^{ \pm} f\left(\varrho, \boldsymbol{H}_{0}\right)}{\partial \varrho}=\lim _{\delta \rightarrow 0} \frac{f\left(\varrho \pm \delta, \boldsymbol{H}_{0}\right)-f\left(\varrho, \boldsymbol{H}_{0}\right)}{\delta} \\
& \frac{\partial^{ \pm} f\left(\varrho, \boldsymbol{H}_{0}\right)}{\partial \boldsymbol{H}_{0, x}}=\lim _{\varepsilon \rightarrow 0} \frac{f\left(\varrho, \boldsymbol{H}_{0} \pm \varepsilon \boldsymbol{i}\right)-f\left(\varrho, \boldsymbol{H}_{0}\right)}{\varepsilon} \\
& \frac{\partial^{ \pm} f\left(\varrho, \boldsymbol{H}_{0}\right)}{\partial \boldsymbol{H}_{0, y}}=\lim _{\varepsilon \rightarrow 0} \frac{f\left(\varrho, \boldsymbol{H}_{0} \pm \varepsilon j\right)-f\left(\varrho, \boldsymbol{H}_{0}\right)}{\varepsilon} \\
& \frac{\partial^{ \pm} f\left(\varrho, \boldsymbol{H}_{0}\right)}{\partial \boldsymbol{H}_{0, z}}=\lim _{\varepsilon \rightarrow 0} \frac{f\left(\varrho, \boldsymbol{H}_{0} \pm \varepsilon \boldsymbol{k}\right)-f\left(\varrho, \boldsymbol{H}_{0}\right)}{\varepsilon}
\end{aligned}
$$

where $\boldsymbol{j}, \boldsymbol{j}$ and $\boldsymbol{k}$ are unit vectors along arbitrarily chosen $x, y$ and $z$ axes exist everywhere on $0<\varrho<\varrho_{c}$ and for all $\boldsymbol{H}_{0}$ ([10], Theorem 111). A longer discussion of the properties to be deduced from the convexity of the free energy as a function of $\varrho$ may be found in Fisher [2]. The corresponding consequence of its convexity as a function of $\boldsymbol{H}_{0}$ is that each component of the magnetization of the system exists for almost all $\boldsymbol{H}_{0}$.

\section{Electric Forces and Fields}

We note that except in giving a physical interpretation to our boundary conditions, we have not used, at any stage, the specific fact that we are dealing with magnetic dipoles in magnetic fields. Thus the whole of the mathematical treatment would equally well apply to a mixture of electric charges and dipoles in an electric field constant on the boundary. An unfortunate feature of the boundary conditions (2.13) however is that by Gauss's theorem they insist that the total charge on the whole system be zero. This is unfortunate for we would like to be 
able to prove the existence of the free energy for the case of a small imbalance in the net charge of the system, as did Lieb and Lebowitz for the case of zero applied field.

In constructing the existence proof for the free energy of a system with electrical forces it is clearer to carry through all of the analysis of Section II using the equivalent charge distributions rather than the dipole moment distribution as the charge distribution is simpler to interpret physically.

Acknowledgement. O. Penrose wishes to thank Monsieur L. Motchane for his hospitality at the I.H.E.S., Bures-sur-Yvette when part of this work was done.

\title{
References
}

1. Ruelle, D.: Helv. Phys. Acta 36, 183-197 (1963).

2. Fisher, M.E.: Arch. Rat. Mech. Anal. 17, 377-410 (1964).

3. Onsager, L.: J. Phys. Chem. 43, 189-196 (1939).

4. Fisher, M.E., Ruelle, D.: J. Math. Phys. 7, 260-270 (1966).

5. Dyson, F. J., Lenard, A.: J. Math. Phys. 8, 423-434 (1967).

6. Lebowitz, J. L., Lieb, E.: Phys. Rev. Letters 22, 631 -634 (1969).

7. Courant, R., Hilbert, D.: Methods of mathematical physics, Vol. II. New York: Interscience 1966.

8. Garabedian,P.R.: Partial differential equations. New York: Wiley 1964.

9. Rogosinski, W. W.: Volume and integral. London: Oliver and Boyd 1962.

10. Hardy, G. H., Littlewood, J. E., Polya, G.: Inequalities. Chapter III. Cambridge University Press 1964.

11. Bak, Th. (Ed.): Statistical mechanics: foundations and application, p. 98. New York: W. A. Benjamin 1967.

\author{
O. Penrose \\ E. R. Smith \\ Mathematics Department \\ The Open University \\ Walton Hall \\ Walton, Bletchley, Bucks., U.K.
}

